\title{
EVALUACIÓN DE ESTRATEGIAS DE MANEJO PARA UNA POBLACIÓN EXPLOTADA CON CRECIMIENTO LOGÍSTICO
}

\section{MANAGEMENT STRATEGY EVALUATION (MSE) FOR A HARVESTED LOGISTIC POPULATION}

\author{
Ricardo Oliveros-Ramos ${ }^{1}$ y Jorge Tam ${ }^{2}$
}

\begin{abstract}
Resumen
El manejo sostenible de las pesquerías es todavía un problema abierto. La evaluación de estrategias de manejo (MSE) permite comparar la eficiencia de las diferentes estrategias de manejo en función al cumplimiento de objetivos operacionales propuestos con el fin de asegurar la sostenibilidad. El objetivo del presente trabajo fue realizar un análisis teórico sobre la evaluación de diferentes estrategias de manejo bajo condiciones de incertidumbre, a partir de la descripción de los niveles iniciales de biomasa que pueden ser manejados sosteniblemente (núcleo de viabilidad) $\mathrm{y}$ sus capturas sostenibles asociadas (controles viables). Las capturas fueron modeladas por $Y=(1+\delta)(1+\omega) Y^{*}$, donde $\delta, \omega>0$ son variables aleatorias y $Y^{*}$ es la cuota de captura determinada por cada estrategia de manejo a partir de una estimación de la biomasa total con incertidumbre. Se exploraron diez estrategias de manejo basadas en la teoría de viabilidad: E1) cuota social, E2) $\left.Y_{\max }(B), E 3\right) \mathrm{Y}$ promedio entre $\left.\mathrm{Y}_{\min } \mathrm{y} \mathrm{Y}_{\max }(\mathrm{B}), \mathrm{E} 4\right) \mathrm{Y}$ aleatoria entre $\mathrm{Y}_{\min } \mathrm{y} \mathrm{Y}_{\max }(\mathrm{B}), \mathrm{E}$ ) $\mathrm{F}_{\mathrm{MSY}}$, E6) $Y_{P A}=Y$ precautoria, E7) promedio entre $Y_{\text {pa }}$ y $Y_{\min }$, E8) aleatoria entre $Y_{\text {pa }}$ y $Y_{\min }$. E9) $Y_{\text {viable }}=$ MVY y, E10) promedio entre $Y_{\mathrm{pa}} \mathrm{y}$ MVY. Las estrategias de manejo fueran evaluadas según nueve indicadores; tres asociados a los riesgos de pérdida de sostenibilidad de cada estrategia, cuatro al promedio y variabilidad de las biomasas y capturas obtenidas y dos al tiempo promedio en el que se pesca más que la cuota social y en el que se imponen moratorias. En base a estos indicadores se determinó que con la estrategia E10, que combina el enfoque precautorio con los resultados de viabilidad, se obtienen mejores resultados, tanto a nivel de conservación de la biomasa como de producción de la pesquería.
\end{abstract}

Palabras clave: Teoría de control viable, manejo de pesquerías, modelo logístico con captura, simulación estocástica.

\begin{abstract}
The sustainable management of the fisheries is still an open problem. The Management Strategy Evaluation (MSE) allows the comparison of the performance of different strategies, based on the accomplishment of operational objectives, proposed in order to ensure sustainability. The goal of the present work was to conduct a theoretical analysis on the evaluation of different management strategies under conditions of uncertainty from the description of the initial biomasses that can be managed sustainably (viability kernel) and their related sustainable yields (viable controls). The catches were modeled by $Y=(1+\delta)(1+\omega) Y^{*}$, where $\delta, \omega>0$ are random variables and $Y^{*}$ is the catch quota given by each management strategy from a total biomass estimate with uncertainty. We explored ten management strategies based on viability theory: E1) social quota, E2) $\mathrm{Y}_{\max }(\mathrm{B})$, E3) $\mathrm{Y}$ average between $\mathrm{Y}_{\min }$ and $\mathrm{Y}_{\max }(\mathrm{B})$, E4) $\mathrm{Y}$ random between $\mathrm{Y}_{\min }$ and $Y_{\max }(B)$, E5) $F_{M S Y}$, E6) $Y_{P A}=Y$ precautionary, E7) average between $Y_{\text {pa }}$ and $Y_{\min }$, E8) random between $Y_{p a}$ and $Y_{\text {min }}$. E9) $Y_{\text {viable }}=M V Y$ and, E10) average between $Y_{p a}$ and MVY. Management strategies were evaluated according to nine indicators; three based on risks of sustainability loss, four based on mean and variance of biomasses and catches obtained, and two based on average time when catches were higher than the social quota and when fishery moratoria were imposed. Based on these indicators we determined that strategy E10, which combines precautionary approach and viability results, had best results, both in biomass conservation and fishery production.
\end{abstract}

Key words: Viability theory, fisheries management, discrete logistic with harvest, stochastic simulation. 


\section{Introducción.}

Una estrategia de manejo es definida por Butterworth et al. (1997) como un conjunto de reglas que traducen la información de una pesquería en un mecanismo regulador (e. g. la máxima captura permisible o el máximo esfuerzo de pesca). Idealmente, antes de elegir una estrategia de manejo, uno debería evaluar las alternativas disponibles y clasificarlas de acuerdo a su habilidad para mantener la pesquería sostenible dentro de un contexto de incertidumbre. En una pesquería, las principales fuentes de incertidumbre son: (i) la variabilidad en la dinámica poblacional, (ii) la imprecisión en la evaluación de los stocks y, (iii) la imprecisión en la implementación de las cuotas de captura (Roughgarden \& Smith, 1996).

La metodología de Evaluación de Estrategias de Manejo (MSE, Management Strategy Evaluation) implica un conjunto de procedimientos basados en la simulación para evaluar y comparar estrategias de manejo alternativas (De Lara \& Martinet, 2008). Sainsbury et al. (2000) detallan más específicamente que el enfoque del MSE consiste en definir un conjunto de objetivos operacionales y evaluar, por medio de simulaciones que tomen en cuenta la incertidumbre en el proceso modelado, el rendimiento de las estrategias de manejo alternativas en relación al cumplimiento de los objetivos planteados.

En este contexto, el objetivo del presente trabajo es evaluar mediante el MSE un conjunto de estrategias destinadas a asegurar la sostenibilidad de una población hipotética con crecimiento logístico. Las estrategias consideradas serán definidas en el marco de la teoría de viabilidad (Aubin, 1991; De Lara \& Doyen, 2008). Esta teoría considerará el núcleo de viabilidad, el cual describe las biomasas para las que existe una sucesión de capturas que aseguran su explotación sostenible por tiempo indefinido; y las capturas viables asociadas a cada biomasa en el núcleo de viabilidad, que proporcionan niveles de captura sostenibles. Así, cada estrategia de manejo nos permitirá escoger una cuota de entre todas las posibles para cada biomasa en el núcleo de viabilidad. Estas estrategias serán comparadas también con estrategias clásicas como la del enfoque precautorio del ICES (International Council for the Exploration of the Sea) y la de una mortalidad por pesca constante para alcanzar el máximo rendimiento sostenible (MSY, Maximum Sustainable Yield). Las fuentes de incertidumbre a ser consideradas serán la segunda y la tercera. En ellas se incluye el efecto de la imprecisión en la estimación de las biomasas utilizadas para la asignación de cuotas, los descartes (biomasa muerta devuelta al mar ya sea porque los individuos capturados presentan tamaños menores a los mínimos permitidos o por insuficiente capacidad de bodega), y la capturas no reportadas por encima de la cuota establecida (pesca negra). El horizonte de simulación será de 50 años.

Se hipotetiza que las estrategias precautorias viables aseguran la sostenibilidad en el largo plazo. Se usaron como criterios para la evaluación de las estrategias los riesgos de pérdida de sostenibilidad, el promedio y variabilidad de las biomasas y capturas obtenidas y, el tiempo promedio en el que se pesca más que la cuota social (i.e. desembarque mínimo necesario para que la pesquería pueda seguir funcionando sin pérdidas económicas ni desempleo para los pescadores).

\section{Materiales y métodos. \\ Dinámica poblacional.}

La dinámica poblacional del stock se simuló utilizando un modelo logístico discreto con capturas discretas (Haddon, 2001), que es definido por:

$$
\begin{aligned}
B(t+1) & =f(B(t), Y(t)) \\
& =R B(t)-\frac{R-1}{K} B^{2}(t)-Y(t),
\end{aligned}
$$

donde $B(t)$ es la biomasa de la población en el tiempo $t, Y(\mathrm{t})$ es la captura en el intervalo [t,t+1[. Los parámetros del modelo fueron la tasa de incremento discreto $(R)$ y la capacidad de carga de la población $(K)$. En este trabajo por simplicidad se asumió una población hipotética con $\mathrm{R}=1.5$ año $^{-1}$ (la unidad de tiempo es arbitraria) y $\mathrm{K}=1$ (la capacidad de carga relativa es adimensional).

Control viable.

La población fue manejada en base a puntos de referencia, uno ecológico $\left(\mathrm{B}_{\min }\right)$ y otro socioeconómico $\left(\mathrm{Y}_{\min }\right)$. El punto de referencia ecológico $\mathrm{B}_{\min }$ está asociado a la mínima biomasa necesaria para que la población pueda sobrevivir en condiciones naturales, mientras que el punto de referencia socioeconómico $Y_{\min }$ es la cuota social, definida como el desembarque mínimo necesario para que la pesquería pueda seguir funcionando sin pérdidas económicas ni desempleo para los pescadores. Se consideraron además dos puntos de referencia precautorios, la biomasa precautoria $\mathrm{B}_{\mathrm{pa}}>\mathrm{B}_{\min }$ para la biomasa y la mortalidad por pesca precautoria $\mathrm{F}_{\mathrm{pa}}$, a fin de tomar en cuenta posibles fuentes de incertidumbre no consideradas en la dinámica (e.g. El Niño). Cabe señalar que con el propósito de evitar el síndrome de puntos de referencia móviles (i. e. el gradual declinamiento de las líneas de base de biomasas de peces) (Pauly, 1995) estos puntos de referencia deben ser constantes bajo cualquier escenario ambiental y de incertidumbre.

Se asumieron valores de $\mathrm{B}_{\min }=0.2, \mathrm{Y}_{\min }=0.05$,

$$
\begin{aligned}
& \mathrm{B}_{\mathrm{pa}}=1.5 \mathrm{~B}_{\min } \mathrm{y} F_{\mathrm{pa}}=\frac{2}{3} F_{\mathrm{MSY}}=\frac{R-1}{3}=0.5 \mathrm{año}^{-1} \text {, en } \\
& \text { donde } F_{\mathrm{MSY}}=\frac{R-1}{2}=0.25 \mathrm{año}^{-1} \text { es la mortalidad por }
\end{aligned}
$$


pesca asociada Máximo Rendimiento Sostenible (MSY).

A partir de los puntos de referencia, se definieron los estados deseables para el sistema, que en el contexto de la teoría de viabilidad (Aubin, 1991) se denomina conjunto deseable $\mathbf{D}$ :

$$
\mathbf{D}=\left\{(B, Y) \mid \begin{array}{c}
B_{\min } \leq B \leq K \\
Y \geq Y_{\min }
\end{array}\right\} .
$$

En este contexto, la sostenibilidad se consideró como la habilidad de mantener al sistema dentro del conjunto deseable durante un tiempo indefinido (De Lara et al., 2007).

Definimos el núcleo de viabilidad como el conjunto de todos los estados del stock que pueden ser manejados sosteniblemente. El núcleo de viabilidad $\mathbf{V}(f, \mathbf{D})$ depende de la dinámica $f$ y el conjunto deseable D. Con la dinámica definida en (1) y el conjunto deseable definido en (2) el núcleo de viabilidad es equivalente a $\mathbf{V}(f, \mathbf{D})=\left\{B \mid B_{\min } \leq B \leq K\right\} \quad$ (Oliveros-Ramos \& Tam, 2008). Para cada biomasa $B$ en el núcleo de viabilidad, se define un conjunto de controles viables $\mathbf{Y}(B)$ como el conjunto de todas las capturas que pueden aplicarse sobre $B$ de manera que la población se mantenga dentro del núcleo de viabilidad. En este caso, el conjunto de controles viables es $\mathbf{Y}(B)=\left[Y_{\min }, Y_{\max }(B)\right]$, en donde $Y_{\max }(\mathrm{B})$ es la captura máxima que se puede aplicar sobre la población, definida por $f\left(B, Y_{\max }(B)\right)=B_{\min }$, que para la dinámica considerada en la ecuación (1) es equivalente a $Y_{\max }(B)=f(B, 0)-B_{\min }$. Definiremos también el máximo rendimiento viable (MVY) por $M V Y=Y_{\max }\left(B_{\min }\right)$, es decir, la captura máxima que se puede aplicar sobre una población con biomasa equivalente al punto de referencia ecológico $\mathrm{B}_{\min }$. El MVY tiene la propiedad de que representa un nivel de captura sostenible para cualquier biomasa en el núcleo de viabilidad (Oliveros-Ramos \& Tam, 2008).

Estrategias de manejo.

En este trabajo, las estrategias de manejo son reglas de correspondencia que le asignan a cada nivel del stock (definido en términos de biomasa) una cuota de captura. Se consideraron diez estrategias de manejo: (E1) la cuota social correspondiente al mínimo nivel de pesca necesario para mantener en funcionamiento la pesquería (5\% de la capacidad de carga de la población); (E2) la cuota máxima viable, para biomasas en el núcleo de viabilidad; (E3) cuota promedio entre E1 y E2; (E4) cuota escogida al azar entre E1 y E2; (E5) mortalidad por pesca para el MSY $\left(F_{\mathrm{MSY}}\right)$ constante; (E6) enfoque precautorio del ICES;
(E7) cuota precautoria promedio entre E1 y E6; (E8) cuota precautoria escogida al azar entre E1 y E6; (E9) cuota constante igual al MVY y; (E10) cuota precautoria promedio entre E6 y E9. La definición de las estrategias de manejo se muestra en la Tabla 1. En todas las estrategias se considera la imposición de una moratoria si $B<B_{\min }$. Con respecto a la racionalidad de las estrategias de manejo, la estrategia E1 define la menor captura posible que satisface con los requerimientos socioeconómicos, mientras que la E2 define la mayor captura que el modelo de control viable establece como sostenible. Entre estos dos extremos se encuentran la estrategia E3 que toma el punto valor medio entre ambas y la E4 que elige al azar entre ellas pero tiene el mismo valor esperado que E3. Las estrategias E5 y E6 son usuales en el manejo de pesquerías. Las estrategias E7 y E8 mantienen el mismo sentido que E3 y E4, mostrando un compromiso entre E1 y E6, teniendo ambas el mismo valor esperado. La estrategia E9 plantea la aplicación constante del MVY, un nivel de captura sostenible para cualquier estado del stock dentro del núcleo de viabilidad. Finalmente, la estrategia E10 es similar a la estrategia E7 pero tomando como límite inferior al MVY en vez de a la cuota social. En general, las estrategias estocásticas usadas (E4 y E8) presentan la posibilidad de escoger cualquier nivel de captura dentro de un cierto rango, que es además equivalente al usado para definir estrategias que también son evaluadas (E3 y E7), teniendo además el mismo valor esperado que estas últimas.

Evaluación de las estrategias de manejo.

La Evaluación de Estrategias de Manejo (MSE) fue utilizada para comparar estrategias utilizadas en el manejo pesquero definidas a partir de los resultados del control viable de la población.

Para la evaluación de estrategias de manejo se consideraron dos escenarios por estrategia y nueve indicadores de sostenibilidad. El análisis se realizó mediante simulación de Monte Carlo, con 10000 simulaciones por cada escenario y estrategia. La fuente de incertidumbre se fijó para cada repetición, a fin de reducir los errores en la comparación de las diversas estrategias. Para cada simulación se calculó el promedio y la varianza de las biomasas simuladas para todo el período de estudio, y luego se calculó la media y la desviación estándar de los valores obtenidos en todas las simulaciones a fin de tener un estimador asociado a cada estrategia de manejo. Estos estimadores se utilizaron para definir posteriormente algunos de los indicadores de sostenibilidad. Para realizar las simulaciones se implementó un programa en lenguaje $\mathrm{R}$ versión 2.8 ( $\mathrm{R}$ Development Core Team, 2009). 
Tabla 1. Definición de las estrategias de manejo utilizadas en este trabajo. Se muestra la forma en la que se define la cuota en cada uno de las diez estrategias (E1-E10).

\begin{tabular}{|c|c|c|}
\hline & Estrategia & Definición \\
\hline E1 & $Y^{*}=Y_{\min }$ & Cuota mínima social \\
\hline E2 & $Y^{*}=Y_{\max }(B)$ & $\begin{array}{l}\text { Cuota viable máxima, que depende del estado de la } \\
\text { población. }\end{array}$ \\
\hline E3 & $Y^{*}=0.5\left(Y_{\min }+Y_{\max }(B)\right)$ & $\begin{array}{c}\text { Cuota promedio entre la cuota mínima social y la cuota } \\
\text { viable máxima. }\end{array}$ \\
\hline E4 & $Y^{*} \sim \operatorname{Uniforme}\left(Y_{\min }, Y_{\max }(B)\right)$ & $\begin{array}{l}\text { Cuota aleatoria viable, con distribución uniforme entre } \\
\text { las capturas viables. }\end{array}$ \\
\hline E5 & $Y^{*}=\operatorname{máx}\left(F_{\mathrm{MSY}} B, Y_{\min }\right)$ & Máximo rendimiento sostenible \\
\hline E6 & $Y^{*}= \begin{cases}Y_{\min } & B<B_{p a} \\
F_{p a} B & B \geq B_{p a}\end{cases}$ & Cuota precautoria del ICES. \\
\hline E7 & $Y^{*}=\left\{\begin{array}{cl}Y_{\min } & B<B_{\mathrm{pa}} \\
0.5\left(Y_{\min }+F_{\mathrm{pa}} B\right) & B \geq B_{\mathrm{pa}}\end{array}\right.$ & Cuota precautoria viable media. \\
\hline E8 & $Y^{*}=\left\{\begin{array}{cl}Y_{\min } & B<B_{\mathrm{pa}} \\
\text { Uniforme }\left(Y_{\min }, F_{\mathrm{pa}} B\right) & B \geq B_{\mathrm{pa}}\end{array}\right.$ & $\begin{array}{l}\text { Cuota precautoria viable aleatoria, con distribución } \\
\text { uniforme entre la cuota social y la cuota precautoria } \\
\text { viable. }\end{array}$ \\
\hline E9 & $Y^{*}=Y_{\text {viable }}=M V Y$ & $\begin{array}{l}\text { Cuota máxima viable (sostenible), independiente del } \\
\text { estado de la población. }\end{array}$ \\
\hline E10 & $Y^{*}=\left\{\begin{array}{cl}Y_{\min } & B<B_{\mathrm{pa}} \\
0.5\left(\mathrm{MVY}+F_{\mathrm{pa}} B\right) & B \geq B_{\mathrm{pa}}\end{array}\right.$ & Cuota precautoria viable media con respecto al MVY. \\
\hline
\end{tabular}

\section{Escenarios.}

Tomando en cuenta el estado inicial del stock $B_{0}$, se usaron dos escenarios plausibles: (S1) escenario saludable, en el que la población se encuentra en un nivel de biomasa superior a la biomasa precautoria $\mathrm{B}_{\mathrm{pa}}$ $\mathrm{y}$, (S2) escenario de recuperación, asumiendo que una población acaba de comenzar un proceso de recuperación luego de colapsar $B<B_{\min }$ ), por lo que la biomasa inicial es inferior a $B_{\text {min }}$. Para el primer escenario se asumió $B_{0}=2 B_{\min }$ y para el segundo $B_{0}=0.6 B_{\text {min }}$.

Fuentes de incertidumbre.

Se consideró la imprecisión en la estimación del tamaño del stock, el efecto de los descartes y la pesca negra como fuentes de incertidumbre. La cuota $Y^{*}(t)$ fue estimada según las reglas dadas por cada estrategia, utilizando un estimado de la biomasa $(\beta)$, la cual estuvo modelada por una variable aleatoria con distribución normal con media igual a la biomasa real (B) y desviación estándar igual al 10\% del tamaño del stock:
$\beta(t)=B(t)(1+\sigma(t))$, donde $\sigma(\mathrm{t})$ es el error en la evaluación del stock en el tiempo $t$, y $\{\sigma(t)\}_{t=1}^{T}$ es una familia de variables aleatorias independientes con distribución normal, con media $\mu_{\sigma}=0 \quad$ y varianza $\sigma_{\sigma}^{2}=0.01$

Para la incertidumbre en las capturas, la proporción de descarte $\delta$ y de la pesca negra $\omega$ fueron modeladas por dos familias de variables aleatorias independientes con distribución lognormal $\{\delta(t)\}_{t=1}^{T} \mathrm{y}$ $\{\omega(t)\}_{t=1}^{T}$, donde $T=50$ es el horizonte de simulación.

Así, la captura durante el periodo $[t, t+1[$ fue modelada por

$$
Y(t)=(1+\delta(t))(1+\omega(t)) Y^{*}(t),
$$

donde $Y^{*}(t)=Y^{*}(\beta(t))$ es el valor de la cuota de captura para el tiempo $t$, dada a partir de la estimación de biomasa $\beta(\mathrm{t})$. Para el caso del descarte se asumió una media $\mu_{\delta}=0.10$ y varianza $\sigma_{\delta}^{2}=0.09$, y para la 
pesca negra se asumió una media $\mu_{\omega}=0.05$ y varianza $\sigma_{\omega}^{2}=0.04$.

Sistema de indicadores.

Para evaluar la eficiencia de cada una de las estrategias de manejo se definieron nueve indicadores. Cuatro de ellos estuvieron asociados a los niveles de biomasa $\left(B^{(1)}, B^{(2)}\right)$ y captura $\left(Y^{(1)}, Y^{(2)}\right)$ y fueron estimados, teniendo en cuenta su valor promedio y su variabilidad, a partir de la media $\left(\mu_{B}^{*}, \mu_{Y}^{*}\right)$ y la desviación estándar $\left(\sigma_{B}^{*}, \sigma_{Y}^{*}\right)$ del promedio de las biomasas y capturas de los últimos 30 años del horizonte de simulación. Para esto se consideraron 20 años como periodo de estabilización de la estrategia (spin up). Otros tres indicadores estuvieron relacionados con el riesgo de perder la sostenibilidad del stock y fueron estimados a partir del número de simulaciones en las que la población no se mantiene viable durante los primeros 20 años $\left(r_{\text {ini }}^{*}\right)$, con respecto a los últimos 30 años $\left(r_{\text {min }}^{*}\right)$ y, en los que la población se mantiene por encima de los niveles precautorios en los últimos 30 años de simulación $\left(r_{\mathrm{pa}}^{*}\right)$. Se consideraron también dos indicadores relacionados a la fracción de tiempo promedio en que es necesario pescar cantidades menores o iguales a la cuota social $\left(t_{\text {min }}^{*}\right)$ e imponer moratorias $\left(t_{\text {veda }}^{*}\right)$, dada cada una de las estrategias consideradas. La definición de los indicadores se muestra en la Tabla 2.

Para cada estrategia se consideraron diferentes realizaciones de $\{\sigma\}_{0}^{T},\{\delta\}_{0}^{T},\{\omega\}_{0}^{T}$ para las fuentes de incertidumbre, donde $s$ es tiempo para la estabilización de la estrategia, $N$ es el número de simulaciones de Montecarlo realizadas y $\mathrm{T}=50$ es el horizonte de simulación.

\section{Resultados y discusión.}

Evaluación de las estrategias de manejo.

La estrategia E1, equivalente a la cuota social, fue sostenible en ambos escenarios. En en ambos casos se obtuvieron riesgos iguales a 0 excepto en el periodo inicial del escenario de recuperación donde todas las estrategias tuvieron asociadas un riesgo de $100 \%$ $\left(r_{\text {ini }}^{*}=1\right.$, ver Tabla 3$)$. Sin embargo no hubo necesidad de imponer vedas $\left(t_{\text {veda }}^{*} \approx 0\right)$ y se aseguró la biomasa

Tabla 2. Definición de los indicadores de sostenibilidad utilizados en este trabajo.

\begin{tabular}{|c|c|}
\hline Indicador & Definición \\
\hline$B^{(1)}=\frac{\mu_{B}^{*}}{B_{\min }}$ & $\begin{array}{l}\text { Media de la biomasa promedio durante el horizonte de } \\
\text { simulación estandarizada por el punto de referencia } \\
\text { ecológico (biomasa mínima del stock) }\end{array}$ \\
\hline$B^{(2)}=\frac{\sigma_{B}^{*}}{\mu_{B}^{*}}$ & $\begin{array}{l}\text { Variación media de la biomasa promedio durante el } \\
\text { horizonte de simulación estandarizada por la media de la } \\
\text { biomasa promedio. }\end{array}$ \\
\hline$B^{(1)}=\frac{\mu_{Y}^{*}}{Y_{\min }}$ & $\begin{array}{l}\text { Media de la captura promedio durante el horizonte de } \\
\text { simulación estandarizada por el punto de referencia socio- } \\
\text { económico (captura mínima, cuota social) }\end{array}$ \\
\hline$r_{\mathrm{ini}}^{*}=1-\frac{1}{N} \sum_{n=1}^{N} \prod_{t=0}^{s} 1_{\left\{B \geq B_{\min }\right\}}\left[B\left(t, \sigma_{n}(t), \delta_{n}(t), \omega_{n}(t)\right)\right]$ & $\begin{array}{l}\text { Riesgo de colapso de la pesquería durante la estabilización } \\
\text { de la estrategia. }\end{array}$ \\
\hline$r_{\min }^{*}=1-\frac{1}{N} \sum_{n=1}^{N} \prod_{t=s+1}^{T} 1_{\left\{B \geq B_{\min }\right\}}\left[B\left(t, \sigma_{n}(t), \delta_{n}(t), \omega_{n}(t)\right)\right]$ & $\begin{array}{l}\text { Riesgo de colapso de la pesquería después de la } \\
\text { estabilización de la estrategia. }\end{array}$ \\
\hline$r_{\mathrm{pa}}^{*}=1-\frac{1}{N} \sum_{n=1}^{N} \prod_{t=0}^{s} 1_{\left\{B \geq B_{\mathrm{pa}}\right\}}\left[B\left(t, \sigma_{n}(t), \delta_{n}(t), \omega_{n}(t)\right)\right]$ & Riesgo de pérdida de la precautoriedad de la pesquería. \\
\hline$t_{\min }^{*}=\frac{1}{N T} \sum_{n=1}^{N} \sum_{t=1}^{T} 1_{\left\{Y \leq Y_{\min }\right\}}\left[Y\left(t, B(t), \sigma_{n}(t)\right)\right]$ & $\begin{array}{l}\text { Fracción del tiempo en que se pesca menos que la cuota } \\
\text { social durante el horizonte de simulación. }\end{array}$ \\
\hline
\end{tabular}


precautoria $\left(r_{\mathrm{pa}}^{*}=0\right)$ para ambos casos. Las biomasas mostraron una tendencia creciente, estabilizándose en niveles cercanos a la capacidad de carga en ambos escenarios (Figuras 1 y 3 ). Las cuotas promedio fueron constantes e iguales a la captura mínima (Figuras 2 y 4). Sin embargo, aunque E1 aseguró la sostenibilidad del stock, ésta no fue óptima desde el punto de vista económico, pues la pesquería funcionó sólo para no tener pérdidas.

La estrategia E2, que asignó una cuota igual a la captura viable máxima para cada estado del stock, no fue sostenible en ninguno de los escenarios considerados, obteniendo en ambos casos riesgos del $100 \%$ para todos los indicadores (Tabla 3). En esta estrategia, a pesar de que las cuotas fueron sostenibles según los resultados de viabilidad, la captura máxima llevó, en el paso de tiempo siguiente, al stock a la biomasa mínima, siendo muy susceptible a la incertidumbre, tal como se observó en la dispersión de

los resultados de las simulaciones (Figuras 1 y 3). Aunque las biomasas en promedio se mantuvieron por encima de $B_{\min }$, no se aseguró la biomasa precautoria $\left({ }^{r_{\mathrm{pa}}^{*}}=1\right)$ en ningún escenario, apreciando además que en el escenario más pesimista (S2) siempre existió la posibilidad de que el stock colapse nuevamente bajo esta estrategia. Las cuotas promedio estuvieron por encima de la captura mínima (Figuras 2 y 4), sin embargo, bajo una periódica imposición de moratorias $\left(t_{\text {veda }}^{*} \approx 0.5\right.$, ver Tabla 3 ) lo que no sería en absoluto recomendable, debido a que las relativamente altas capturas de un año se compensaron con una veda al año siguiente. Esto afectaría de manera importante a la pesquería, al tener que mantenerse los barcos sin posibilidad de uso. Las cuotas mostraron una alta variabilidad en ambos escenarios ( $Y^{(2)}>1$, Tabla 3), debido a la recurrencia de vedas, situación que no es deseable.

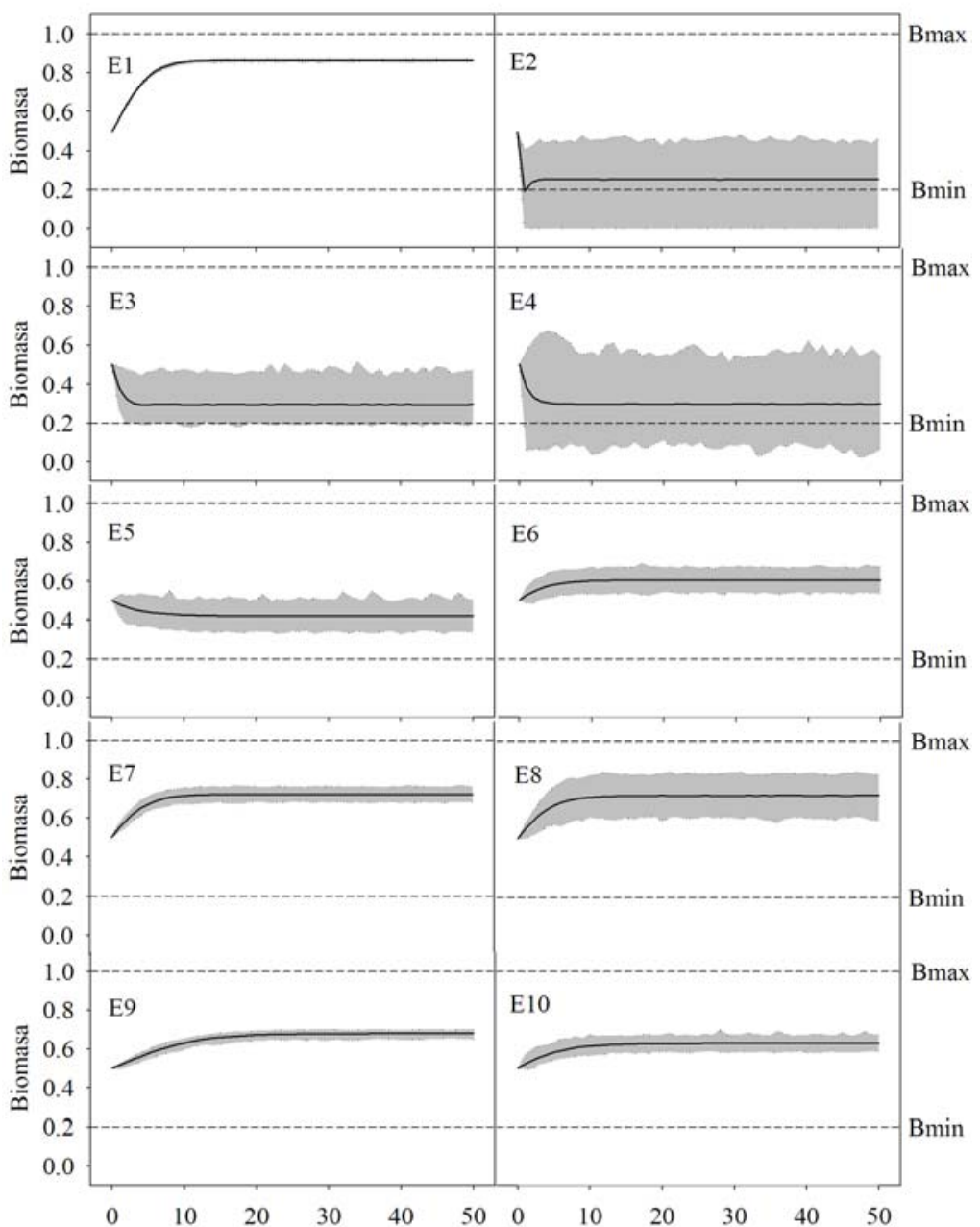

Figura 1. Trayectoria esperada de la biomasa del stock bajo cada una de las estrategias de manejo evaluadas (E1-E10) para el escenario del stock saludable (S1). La zona gris se encuentra entre los límites máximo y mínimo de todas las trayectorias simuladas $(\mathrm{n}=10000)$.
La estrategia E3 representó un intermedio entre las estrategias E1 y E2. Esta estrategia no fue sostenible en ambos escenarios, obteniendo en ambos casos riesgos cercanos al 100\% (Tabla 3). Las biomasas en promedio se mantuvieron por encima de $B_{\min }$, estabilizándose al alrededor del $30 \%$ de la capacidad de carga (Figuras 1 y 3), aunque en ningún escenario se logró asegurar capturas fueron superiores a las obtenidas con las estrategias anteriores, en un nivel casi $80 \%$ por encima de la cuota social ( $Y^{(1)}=1.78$, Tabla 3 ), motivo por el cual se impusieron vedas durante aproximadamente el $16-20 \%$ del periodo (Tabla 3).

La estrategia E4 asignó la cuota de manera estocástica, aunque tuvo un valor esperado equivalente al de la estrategia E3. Esta estrategia no fue sostenible en ninguno de los escenarios considerados, obteniendo en ambos casos riesgos cercanos al $100 \%$ para todos los indicadores (Tabla 3). Los indicadores de la estrategia E4 fueron similares a los de la estrategia E3, diferenciándose principalmente en los indicadores asociados a la varianza de las biomasas y capturas $\left(B^{(2)}, Y^{(2)}\right)$. Esto debido a la elección aleatoria de las cuotas dentro de los controles viables, lo cual aumentó la la biomasa precautoria $\left({ }^{r^{*}}=1\right)$. Las 


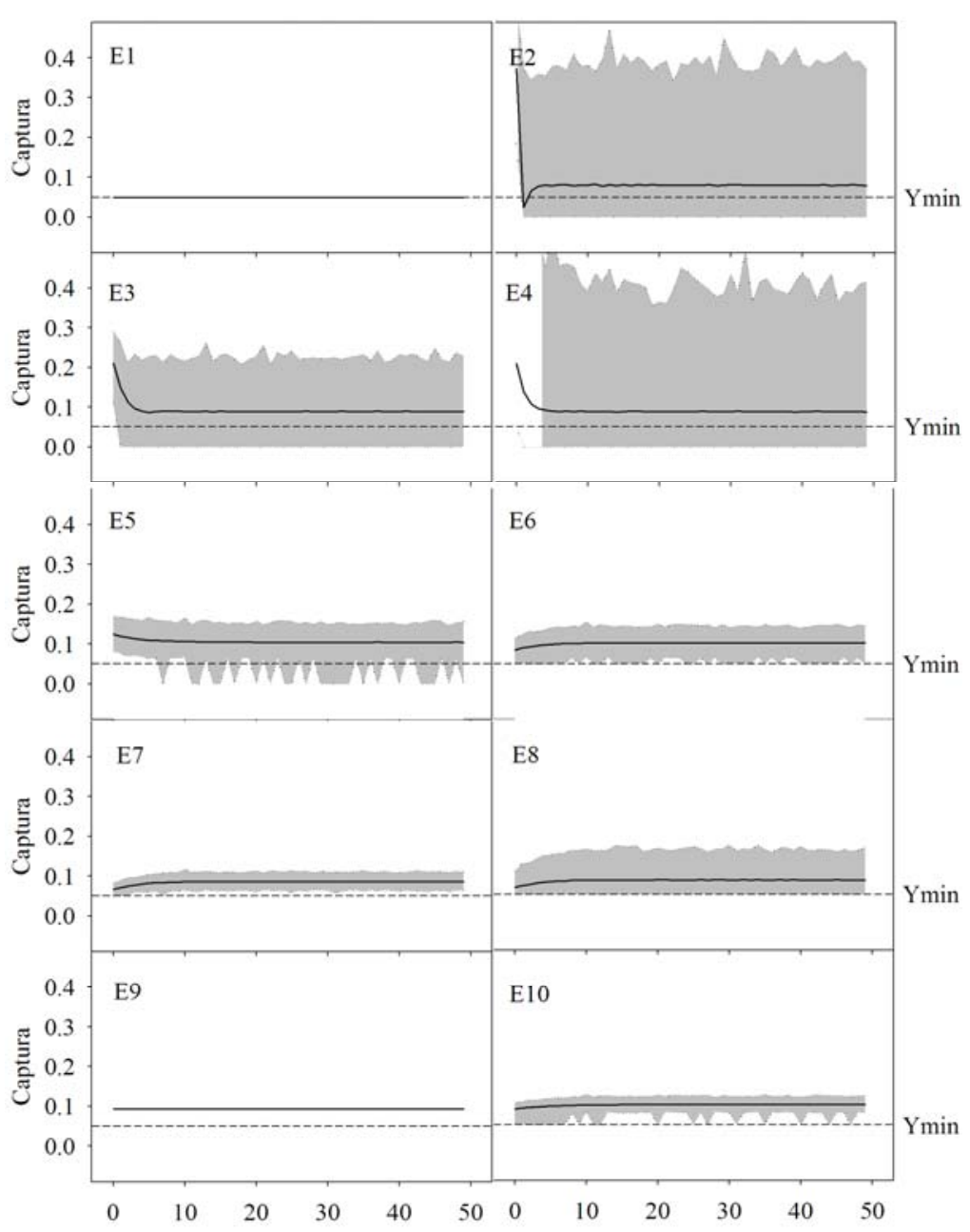

Figura 2. Trayectoria esperada de la cuota para el stock bajo cada una de las estrategias de manejo evaluadas (E1-E10) para el escenario del stock saludable (S1). La zona gris se encuentra entre los límites máximo y mínimo de todas las trayectorias simuladas $(n=10000)$.

variabilidad. Se diferenciaron también de E3 en que el funcionamiento de la pesquería con la cuota social y la imposición de moratorias fue más frecuente (Tabla 3).

La estrategia E5 corresponde a la mortalidad por pesca para alcanzar el MSY, y fue sostenible en ambos escenarios. En ambos casos se obtuvieron riesgos iguales a 0 , excepto para el de preservación de la biomasa precautoria que estuvo entre el 23\% y $25 \%$ (Tabla 3). La biomasa en promedio se estabilizó en el $50 \%$ de la capacidad de carga tal como se esperaba (Figuras 1 y 3), y las cuotas fueron aproximadamente el doble de la cuota mínima (Tabla 3). La frecuencia de capturas mínimas y moratorias fue de $0 \%$ en el escenario saludable y del $5 \%$ en el escenario de recuperación (Tabla 3 ).

La estrategia E6, que asignó cuotas precautorias, fue sostenible en ambos escenarios, obteniendo en ambos casos riesgos iguales a 0 . Con esta estrategia no hubo necesidad de imponer vedas ni pescar la cuota social en el escenario saludable (S1), aunque en el escenario de recuperación (S2) la frecuencia de ambas fue de $4 \%$ y $8 \%$, respectivamente (Tabla 3). Las biomasas mostraron una tendencia creciente, estabilizándose en niveles cercanos al $60 \%$ de la capacidad de carga en ambos escenarios (Figuras 1 y 3). Las cuotas promedio y su variabilidad fueron muy similares a las obtenidas con la E5, pero con el beneficio de asegurar la biomasa precautoria. Considerando en particular el escenario saludable, esta estrategia se presentó como apropiada desde el punto de vista económico, pues no requirió de imposición de moratorias y siempre se pudieron dar cuotas por encima de la cuota social.

La estrategia E7 representa un intermedio entre las estrategias E1 y E6. Esta fue sostenible en ambos escenarios, obteniéndose en ambos casos riesgos iguales a 0 . Con esta estrategia tampoco hubo necesidad de imponer vedas ni pescar la cuota social en el escenario saludable (S1), y en el escenario de recuperación (S2) se obtuvieron casi los mismos resultados que con E6 (Tabla 3). Las biomasas mostraron una tendencia creciente $\mathrm{y}$ fueron superiores a las de E6, estabilizándose en niveles superiores al $70 \%$ de la capacidad de carga en ambos escenarios (Figuras 1 y 3 ). Las cuotas promedio y su variabilidad fueron inferiores a las obtenidas con la estrategia anterior aunque superiores a la cuota social ( $Y^{(1)}=1.72$, Tabla 3 ) y similares a las obtenidas con la estrategia de maximización de la captura, E2. Se puede afirmar que los resultados de esta estrategia son muy similares a las de E6 en términos de conservación, favoreciendo a la preservación de biomasa por sobre la pesquería.

La estrategia E8 asignó la cuota estocásticamente, con un valor esperado equivalente al de la estrategia E7. Los indicadores de la estrategia E8 fueron similares a los de la estrategia E7, diferenciándose principalmente en los indicadores asociados a la varianza de las biomasas y capturas $\left(B^{(2)}, Y^{(2)}\right)$, tal como entre E3 y E4. Sin embargo, en este caso, no se diferenciaron en la frecuencia de imposición de vedas y cuotas mínimas, debido al carácter precautorio que ambas comparten (Tabla 3). 
La estrategia E9 asignó cuotas iguales al MVY, que como se mencionó anteriormente fueron sostenibles para todo stock en el núcleo de viabilidad. Esta estrategia fue sostenible en ambos escenarios, obteniendo riesgos iguales a 0 , salvo en el escenario de recuperación (S2) en donde hubo un riesgo menor al $2 \%$ de no mantener las biomasas mínima o precautoria. Las biomasas mostraron una tendencia creciente, estabilizándose en niveles cercanos al 70\% de la capacidad de carga en ambos escenarios (Figuras 1 y 3), por encima de la biomasa de equilibrio de la estrategia precautoria E6. Las cuotas promedio y su variabilidad fueron superiores a los obtenidos con la estrategia precautoria, con la ventaja de no mostrar variabilidad por ser constante la cuota.

La estrategia E10 representó un intermedio entre las estrategias E6 y E9, siendo similar a E7 pero tomando en cuenta el MVY como cota inferior para cuota. Fue sostenible en ambos escenarios, obteniendo en ambos casos riesgos iguales a 0 . Con esta estrategia tampoco hubo necesidad de imponer vedas ni pescar la cuota social en el escenario saludable, y en el escenario de recuperación se obtuvieron también casi los mismos resultados que con E6 (Tabla 3). Las biomasas mostraron una tendencia creciente y fueron superiores a las de E6, estabilizándose en niveles superiores al $60 \%$ de la capacidad de carga en ambos escenarios (Figuras 1 y 3), con una biomasa de equilibrio mayor a la obtenida con E6 pero inferior a la obtenida con E7. Las cuotas promedio fueron ligeramente inferiores a las obtenidas con la estrategia precautoria E6, pero mostraron una menor variabilidad.

En comparación a E9, las cuotas asignadas fueron mayores y con riesgos menores en el escenario de recuperación. Las frecuencias de vedas y cuotas mínimas fueron similares a las de todas las estrategias precautorias (Tabla 3 ).

Sistema de indicadores.

En la Figura 5 se muestra un resumen de los indicadores evaluados para cada una de las estrategias y escenarios. Los dos indicadores asociados a cada variable (biomasa y captura) se combinaron para tener en cuenta su magnitud relativa y su variabilidad. Así, la zona gris representa la región en la cual la estrategia respondió en promedio con al menos el doble del valor del punto de referencia $\left(B_{\min }, Y_{\min }\right)$ y presentó además una baja variabilidad (menor al $40 \%)$. En teoría, todas las estrategias dentro del área definida como aceptable y con riesgos mínimos fueron aplicables, y se pudieron escoger en función al compromiso que se decidió entre conservación de biomasa y los niveles de pesca. Las estrategias de tipo precautorio tuvieron en común la minimización de los riesgos, pero la estrategia E10, que consideró además los resultados de viabilidad, mostró un mejor desempeño en la conservación de biomasas que el enfoque precautorio simple, $\mathrm{y}$ dando cuotas muy similares.

El siguiente paso será definir indicadores globales, basados en la teoría de la decisión multiatributos y multiobjetivos. Sin embargo, no será sencillo construir un indicador global, pues no es posible definir unívocamente un indicador que resuma el sistema de indicadores, ya que éste dependerá de los diferentes objetivos de manejo, y se le podría estar dando mayor peso a algún indicador en particular. Por otro lado algunos de los indicadores pueden estar correlacionados, ya que hay una relación en la forma en que están definidos, como en el caso de los riesgos definidos por 


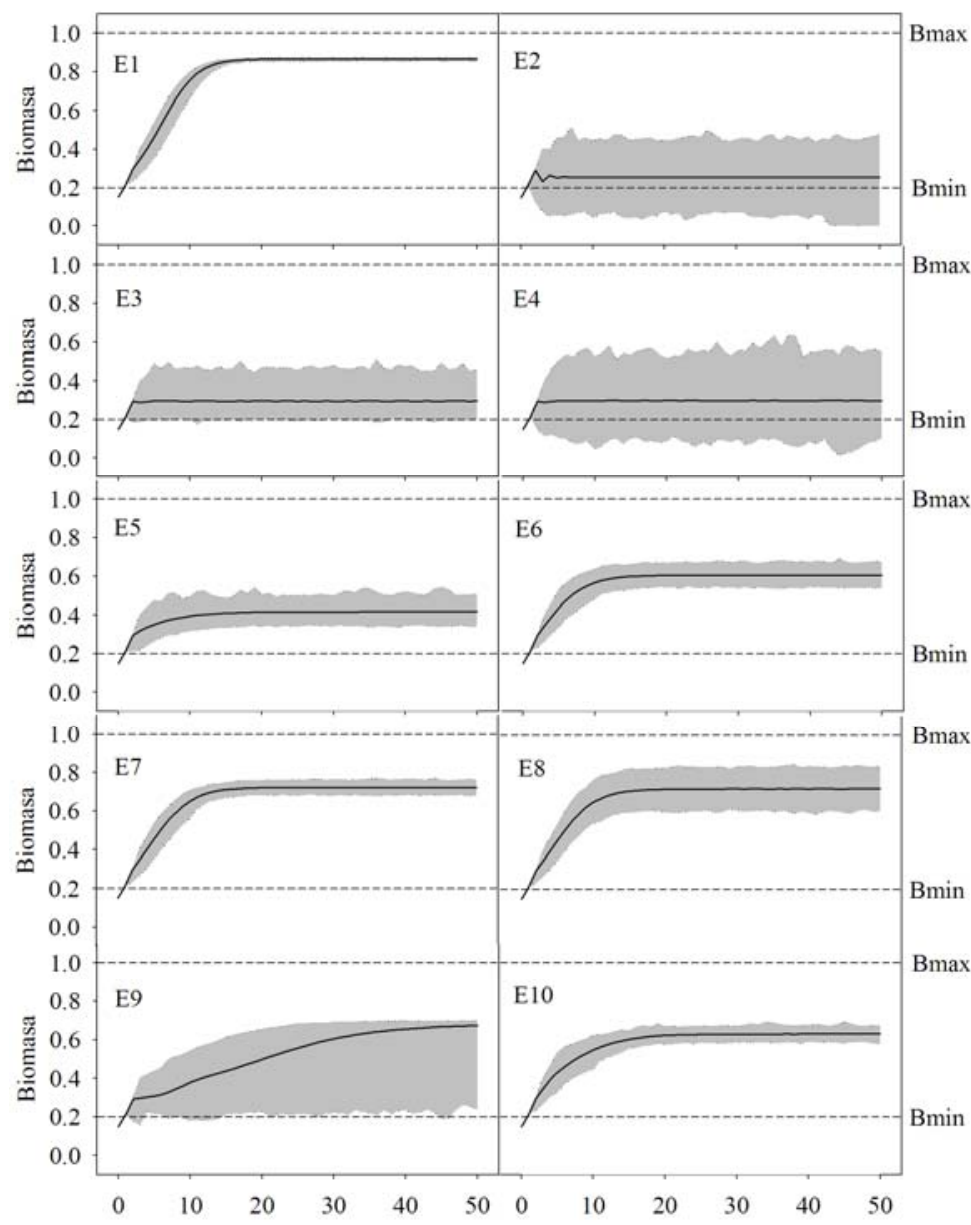

Figura 3. Trayectoria esperada de la biomasa del stock bajo cada una de las estrategias de manejo evaluadas (E1-E10) para el escenario del stock en recuperación (S2). La zona gris se encuentra entre los límites máximo y mínimo de todas las trayectorias simuladas $(\mathrm{n}=10000)$.

puntos de referencia de biomasas y el indicador de abundancia $B^{(1)}$.

\section{Resultados y discusión.}

Las estrategias de manejo que consideraron el enfoque precautorio (E6, E7, E8, E10) fueron robustas a la incertidumbre, minimizando los riesgos de conservación de la biomasa, tanto mínima como precautoria.

La mejor estrategia entre las evaluadas fue la E10, que mostró un mejor desempeño en la conservación de biomasas que las del enfoque precautorio y dió valores de cuotas similares. La estrategia E9 basada en el MVY mostró un muy buen desempeño a pesar de no ser precautoria.

Se recomienda aplicar el MSE como medio de evaluación de estrategias de pesca de los principales recursos pesqueros. Es importante incluir también la incertidumbre proveniente de variables ambientales en la dinámica poblacional, a fin de tomar en cuenta una importante fuente de variabilidad, en especial en el
Norte del Ecosistema de la Corriente de Humboldt. Finalmente, será necesario aplicar metodologías de análisis de decisión para ordenar las estrategias usando indicadores agregados.

\section{Agradecimientos.}

Se agradece a Michel de Lara (CERMICS, Francia) y a Eladio Ocaña (IMCA, Perú) por sus discusiones sobre teoría de viabilidad, y a Arnaud Bertrand por el apoyo financiero del IRD (Francia) para la presentación de este trabajo en el IV Congreso Internacional de Matemática Aplicada y Computacional. Así mismo se agradece a dos revisores anónimos por sus valiosas sugerencias y observaciones que permitieron mejorar la claridad y precisión del trabajo.

\section{Literatura citada.}

Aubin J.P. 1991. Viability Theory. Birkhauser, Boston.

Butterworth D. S., Cochrane K. L. \& Oliveira, J. A. A. D. 1997. Management procedures: a better way to manage fisheries? the South African experience. In E. K. Pikitch, D. D. H. and Sissenwine, M. P., editors, Global Trends: Fisheries Management, pages 83-90. American Fisheries Society Symposium 20.

De Lara M., Doyen L., Guilbaud T. \& Rochet M.-J. 2007. Is a management framework based on spawning-stock biomass indicators sustainable? A viability approach. ICES J. Mar. Sci. 64(4):761-767.

De Lara M. \& Doyen L. 2008. Sustainable Management of Natural Resources. Mathematical Models and Methods. Springer-Verlag, Berlin, 2008.

De Lara M. \& Martinet V. 2008. Multi-Criteria Dynamic Decision Under Uncertainty: Management Strategy Evaluation, Stochastic Viability Analysis and Beyond. MIFIMA meeting, 12-13 Junio, Parıs. On line: http://cermics.enpc.fr/ delara/exposes/slides_ MathBioSc_14June2008.pdf

Haddon M. 2001. Modelling and quantitative methods in fisheries. Chapman \& Hall/CRC, New York. 407 pp.

Oliveros-Ramos R. \& Tam J. 2008. Viability control model for management of fisheries: The case of peruvian hake Merluccius gayi peruanus. In: IRD. Eastern boundary upwelling ecosystems. Abstracts book. Canary Island, Spain., p. 101. On line: www.euroceans.eu/upwellings/ws/2008_06_LasPalmas/ talks/PL5_OP07_Oliveros_R.pdf. 
Roughgarden J. \& Smith F. 1996. Why fisheries collapse and what to do about it. Proc. Natl. Acad. Sci. USA, 93: 5078-5083.

Sainsbury K. J., Punt A. E. \& Smith A. D. M. 2000. Design of operational management strategies for achieving fishery ecosystem objectives. ICES Journal of Marine Science, 57: 731-741.

Pauly D. 1995. Anecdotes and the shifting baseline syndrome of fisheries. Trends in Ecology and Evolution, 10(10):430.

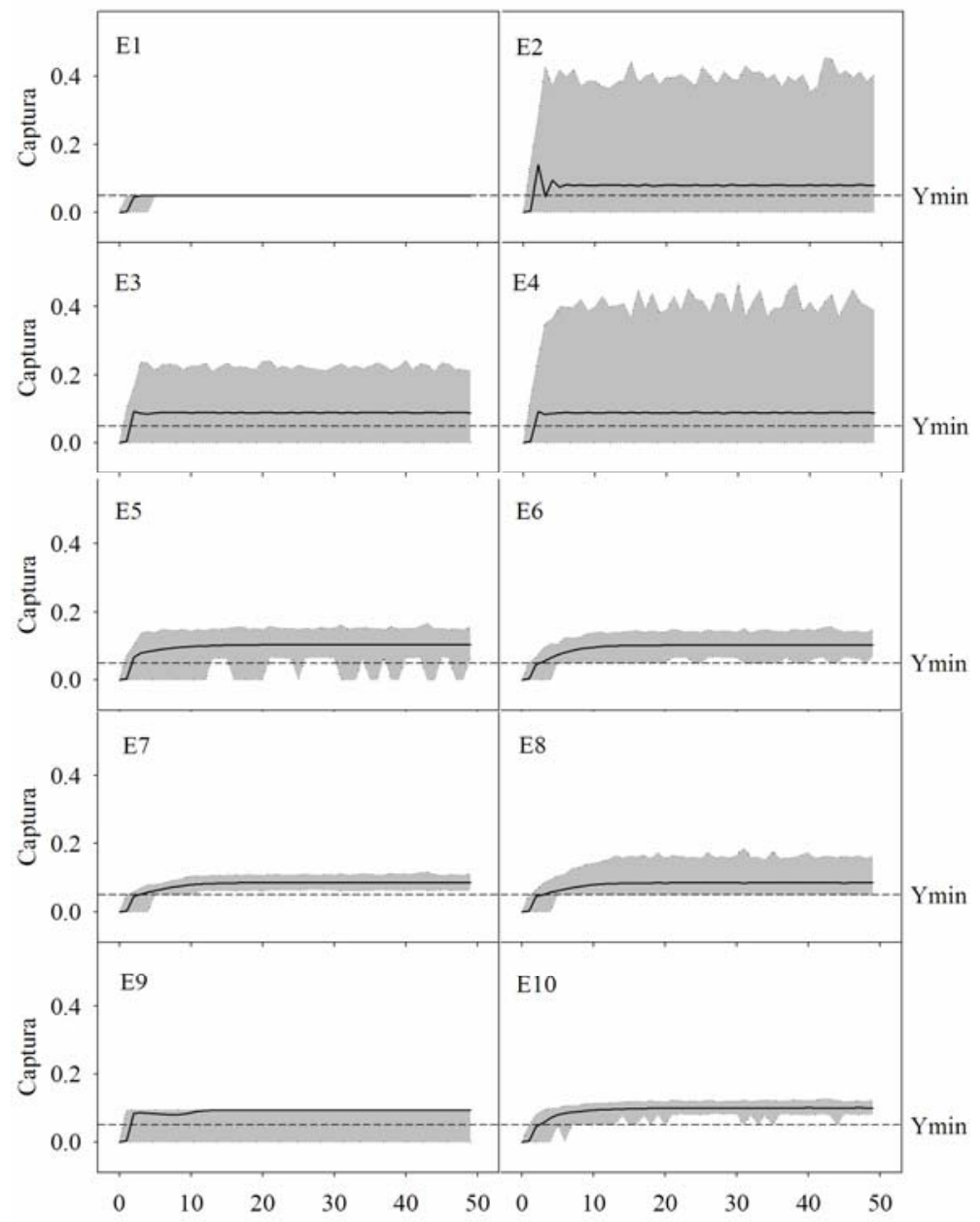

Figura 4. Trayectoria esperada de la cuota para el stock bajo cada una de las estrategias de manejo evaluadas para el escenario del stock en recuperación (S2). La zona gris se encuentra entre los límites máximo y mínimo de todas las trayectorias simuladas $(\mathrm{n}=10000)$. 

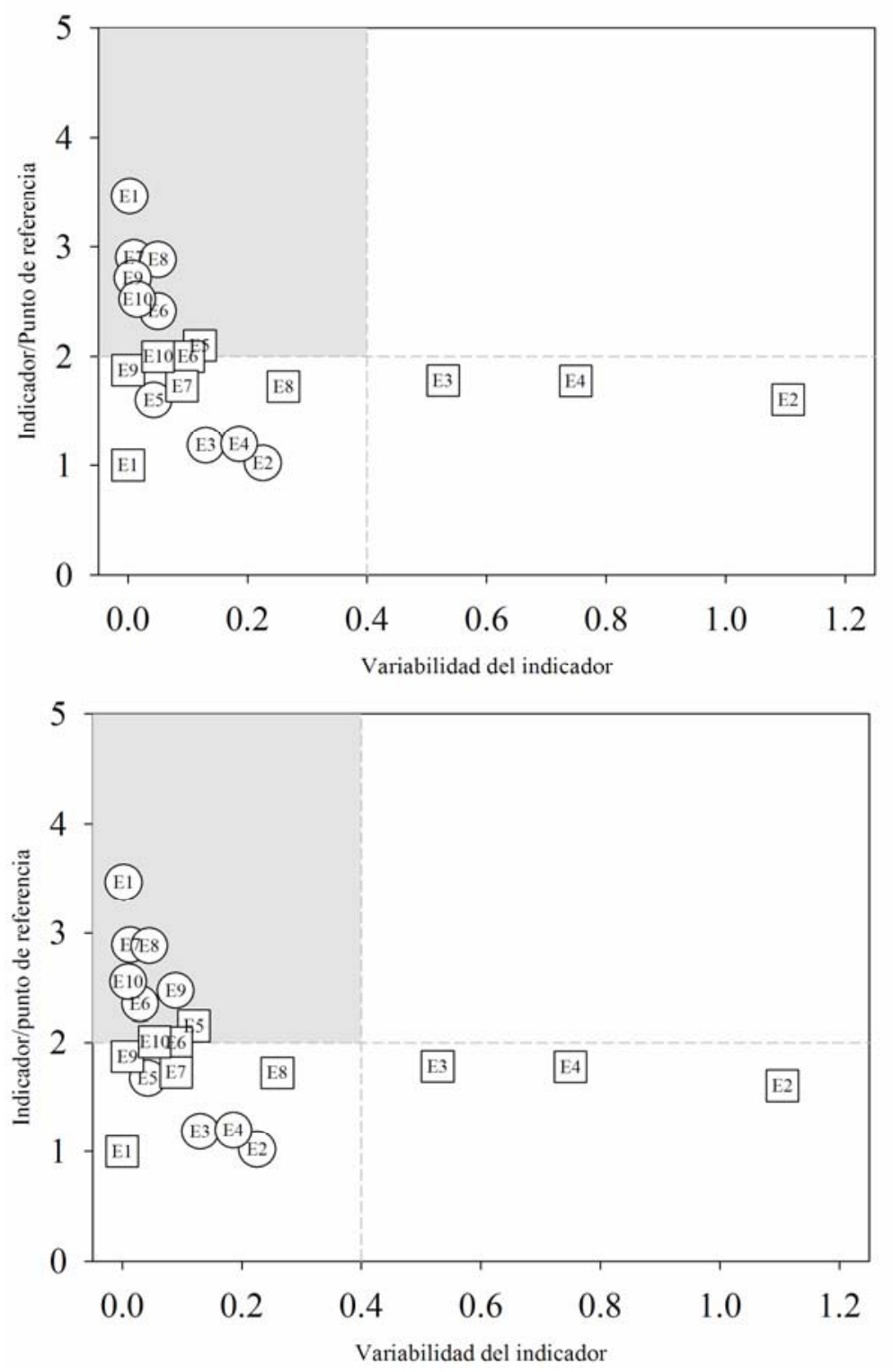

Figura 5. Indicadores de sostenibilidad $\left(B^{(1)}, B^{(2)}, Y^{(1)}, Y^{(2)}\right.$ ) para el escenario del stock saludable S1 (arriba) y en recuperación S2 (abajo). Los indicadores asociados a las capturas se representan con circunferencias y los asociados a la biomasa con cuadrados. El área destacada en gris representa la zona donde los indicadores tuvieron un valor aceptable.

1 Centro de Investigaciones en Modelado Oceanográfico y Biológico Pesquero (CIMOBP), Instituto del Mar del Perú (IMARPE). Esquina Gamarra y Gral. Valle s/n, Chucuito, Callao. roliveros@imarpe.gob.pe

2 Centro de Investigaciones en Modelado Oceanográfico y Biológico Pesquero (CIMOBP), Instituto del Mar del Perú (IMARPE). Esquina Gamarra y Gral. Valle s/n, Chucuito, Callao. jtam@imarpe.gob.pe 